\title{
FREQUENCY DEPENDENCE OF THE EMISSION FROM 2D ARRAY JOSEPHSON OSCILLATORS
}

\author{
P.A.A. Booi and S.P. Benz, \\ National Institute of Standards and Technology, Division 814.03, \\ 325 Broadway, Boulder, CO 80303
}

T. Doderer, D. Hoffmann, J. Schmidt, S. Lachenmann and R.P. Huebener Physikalisches Institut, Lehrstuhl Experimentalphysik II, University of Tübingen, Morgenstelle 14, D-7400 Tübingen, Germany

\begin{abstract}
Coherent emission from two-dimensional arrays of Josephson junctions, coupled to a detector junction through a dc blocking stripline capacitor, was detected over a frequency range from 50 to $210 \mathrm{GHz}$. A power of $0.26 \mu \mathrm{W}$ which is larger than the $0.1 \mu \mathrm{W}$ expected from the RSJ model was detected in a range from 140 to $150 \mathrm{GHz}$. Frequencies where no emission was detected correspond to standing waves in the capacitor when multiples of the half-wavelength match the capacitor length. Low temperature scanning electron microscopy confirmed the presence of standing waves at these frequencies, but also revealed standing waves at other frequencies indicating an impedance mismatch and a possible extension of the standing waves into the array.
\end{abstract}

\section{INTRODUCTION}

Two-dimensional (2D) arrays of $(\mathrm{M} \times \mathrm{N})$ shunted SIS Josephson junctions have been shown to emit coherently over a wide frequency range $[1,2]$. The potential for high power, narrow linewidth, and improved impedance matching makes the application of 2D arrays as voltage tunable high-frequency sources very promising. In $[1,2]$, as in the present experiment, the array emission was coupled to a detector junction through a dc blocking stripline capacitor. Possible mechanisms for this phase locking were proposed. These were based on intrinsic characteristics of the $2 \mathrm{D}$ arrays, such as fluxoid quantization in the four-junction cells and quasilong range interactions between the junctions, and on properties of the detection circuit, such as feedback from the coupling circuit and resonances in the blocking capacitor.

In this paper, we focus on the possible phase-locking mechanisms through quantitative measurements of the emission spectrum in conjunction with low temperature scanning electron microscopy (LTSEM) measurements of the dc and if properties of the circuit. The emission/detection circuit, shown in Fig. 1, and its design considerations have been discussed in detail by Benz and Burroughs [2]. The circuit facilitates the measurement of the frequency dependence of the emitted power in several ways, either by determining the coherent power quantitatively from Shapiro steps induced in the detector current-voltage (I-V) curve, or by determining the total power, both coherent and incoherent power, qualitatively by employing the detector junction as a squarelaw or SIS detector [3]. The SIS detection method is used to examine the whole circuit with LTSEM. The LTSEM technique and results for this experiment are presented in Doderer et al. [4], and the references therein.

Manuscript received August 24, 1992

U.S. Government work not protected by U.S. copyright.

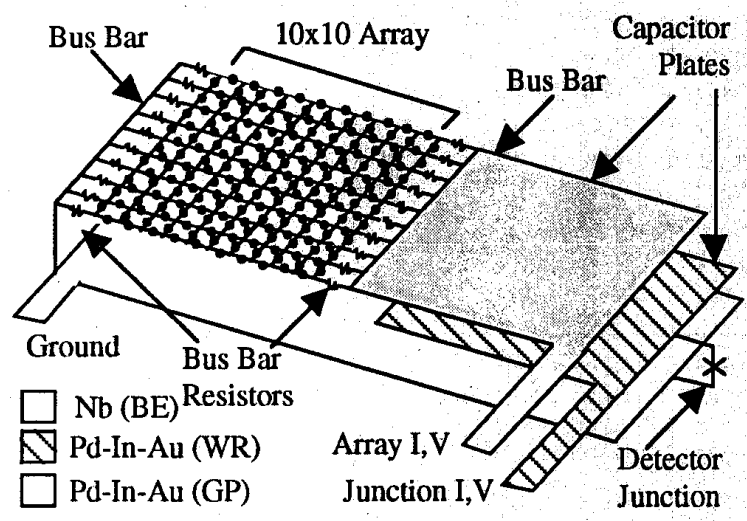

Fig. 1. Schematic layout of the 2D array emission and detection circuit. The array junctions are depicted by black dots. The SiO level between the two top plates defines the dc blocking capacitor.

\section{EXPERIMENTAL}

$\mathrm{The} \mathrm{Nb} / \mathrm{Al}-\mathrm{AlO} \mathrm{O}_{\mathrm{x}} / \mathrm{Nb}$ junctions in the $10 \times 10$ array and the detector junction are identical (the trilayer films are respectively 210,10 and $140 \mathrm{~nm}$ thick, and the junction area is about $18 \mu \mathrm{m}^{2}$ ) with a critical current density of about 550 $\mathrm{A} / \mathrm{cm}^{2}$. The critical current uniformity, as measured from an on-chip 400-junction series array, was within $1 \%$ at one standard deviation. The SIS junctions were designed to be nonhysteretic by using AuIn 2 shunt resistors of $R_{s}=1.1 \Omega$. From the hysteresis in the I-V curves of test junctions on the chip, the capacitance $\mathrm{C}_{\mathrm{J}}$ of the junctions was determined to be $0.4 \mathrm{pF}$; due to the effects of noise on the test junction's I-V curve, this is a lower estimate of the capacitance. Above the characteristic frequency of the junctions, $v_{c}=\mathrm{il}_{\mathrm{g}} / \Phi_{\mathrm{o}} \approx 50$ $\mathrm{GHz}$, the junctions oscillate nearly sinusoidally. The impedances were defined by two SiO levels, where the second $\mathrm{SiO}$ level was used as the dielectric for the capacitor, which was $465 \times 150 \mu \mathrm{m}^{2}\left(\mathrm{C}_{\mathrm{B}}=11.2 \mathrm{pF}\right)$, matching the $150 \mu \mathrm{m}$ array width. The groundplane; which is used to reduce inductances in the array and the coupling circuit, gives rise to a parasitic capacitance between the lower plate (see Fig. 1) of the blocking capacitor and ground which shunts the detector junction, so the effective $\beta_{c}$ of the detector is slightly larger than 1 . The resulting I-V curve hysteresis makes it difficult to quantitatively estimate the 'square-law detected' power, however, this method still reveals useful information about the coupling circuit and the array. To improve the bias 
current uniformity in the array, $M$ resistors, of $0.3 \Omega$ each, connect each side of the array to a $\mathrm{Nb}$ bus bar and the capacitor. The circuits were electrically characterized by fourpoint measurements in a liquid He storage Dewar with the help of a magnetically shielded probe [5]. Filters were used on all lines to reduce high frequency interference.

\section{RESULTS AND DISCUSSION}

Simulations were used to model the detector circuit and match the experimental detector I-V curves, with rf-induced Shapiro steps, at various array emission frequencies. We used the same circuit as in Ref. [1] with the values of the parasitic elements determined from the measured film dimensions for this specific chip. Resonances and occasional subharmonic steps in the measured detector I-V curve could partly be accounted for by using the estimated parasitic elements. In some regions of the experimental detector I-V curve, the resonances obscured the Shapiro steps making an estimation of the power difficult. Figure 2 shows the coherent array output power estimated by matching the experimental and simulated Shapiro steps of the detector as a function of the array frequency (and voltage). Also plotted in this figure is the theoretical coherent power $M^{2} V_{1}^{2} / 8 R_{s}$ generated by a $10 \times 10$ array to a matched load using the RSJ model fundamental voltage amplitude $V_{1}(v)$.

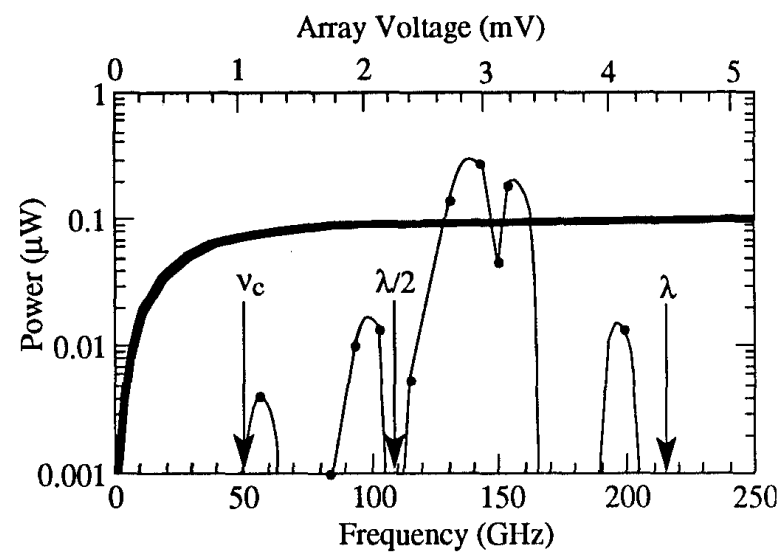

Fig. 2. Coherent emitted array power coupled to the detector junction compared with the array power expected from the RSJ model (fat line). The array voltage $10 \Phi_{0} v$ corresponding to the emission frequency is plotted on the top axis. Data points are denoted by circles and the connecting curve was estimated from induced Shapiro steps in the detector I-V curve.

In the range $140-150 \mathrm{GHz}$, a maximum power of about $0.26 \mu \mathrm{W}$ which is much larger than the $0.1 \mu \mathrm{W}$ maximum power estimated from the RSJ model (see Fig. 2) was detected. This additional power can only partially be accounted for by taking the inductance of the junction's shunt into account [1]. The measured coherent power spectrum in Fig. 2 suggests a power spectrum related to a complex transfer function between the array and detector, with the maximum transfer occurring at a resonance frequency. Other array/detector circuits have been measured $[1,2]$ and the maximum rf power from the arrays always occurred near this same frequency, independent of the dc blocking capacitor length, the shunt resistor values, and whether or not the junctions perpendicular to the array bias were shorted. Since even bare arrays, without a coupling circuit and detector junction, showed a resonance in their I-V curves corresponding to this frequency, we conclude that this frequency is related to a resonance in the array or, more specifically, in the fourjunction cells. Since the junctions perpendicular to the array bias are in the zero-voltage state, a four-junction unit cell can be regarded as a dc SQUID with resonance frequency $\omega=\sqrt{2 / \mathrm{LC}_{\mathrm{J}}}$, in which $\mathrm{L}$ denotes the total cell inductance. Measurements of the stripline inductance for unit cell test structures on the chip give $\mathrm{L}=5.5 \mathrm{pH}$, corresponding to a resonance frequency of $150 \mathrm{GHz}$. Thus, the additional power detected from the array above the RSJ-model power probably results from this resonance, provided it does not exceed the total available power $[1,2]$.

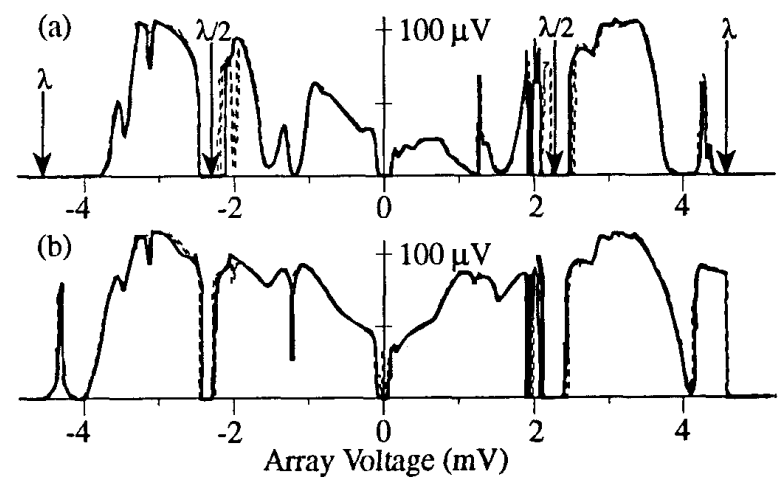

Fig. 3. Detector voltage at a junction bias of (a) $0.9 i_{c}$ and (b) $i_{c}$, as a function of array voltage. The dashed lines denote decreasing array bias. The array voltages have not been corrected for the small voltage across the bus bar resistors (about $2 \times R_{\mathrm{BB}} / 10 \approx 0.06 \Omega$ ) in series with the array.

In Fig. 3, we used the detector junction as a square-law detector for the array emission. The detector voltage is approximately proportional to the square-root of the incident power. In this way, both the coherent and incoherent power coupled from the array is detected. Because of the hysteresis in the detector junction's I-V curve, the power can not be determined quantitatively from Fig. 3 . The asymmetry in this spectrum for positive and negative array biases corresponds to an asymmetry that was observed in the array I-V curve and is probably due to asymmetry in the circuit layout (see Fig. 1). Because of the hysteresis in the detector I-V curve, the clearest traces were obtained when biasing the detector with $i_{d c} \leq i_{c}$.

Power was detected in some regions of Fig. 3 where no coherent emission was detected from Shapiro steps, indicating that incoherent power was measured by the detector. For example, when the array voltage was less than $1 \mathrm{mV}$, that is $v<v_{c}$, no coherent power was detected (see Fig. 2). The nonzero detector voltage in Fig. 3 for these frequencies is therefore a result of incoherent array emission. Regions in 
Fig. 3 where the detector voltage is 0 indicate that no measurable power, either coherent or incoherent, was detected from the array. Two of the zero-coherent-power regions in Fig. 2 correspond to the zero-power regions in Fig. 3 and are designated by arrows. LTSEM measurements [4] showed standing waves in the stripline capacitor, with a fullwavelength standing wave occurring at about $219 \mathrm{GHz}$, suggesting that the zero detected power regions result from total reflected power when half- and full-wavelength standing waves occur in the capacitor.

In order to characterize the zero-power frequency bands and the geometrical standing waves in the blocking capacitor, detailed modeling of the coupling circuit was necessary. The stripline blocking capacitor was divided into 40 segments using inductance and capacitance values from the measured film dimensions, and coupled to the array and detector resistances on either end. The coupling coefficient between the two capacitor plates was estimated to be 0.81 , approximately fitting the LTSEM observations of the fullwavelength standing wave. This is within $5 \%$ of the value $(0.77)$ calculated with a computer program based on Chang's method [6]. The difference between these two coupling coefficient values arises from an uncertainty in the penetration depth of the $\mathrm{Pb}$-In-Au wiring level and groundplane (estimated to be $137 \mathrm{~nm}$ ), since the exact composition of the $\mathrm{Pb}$-alloy is not known. The resulting power transfer function is shown in Fig. 4.



Fig. 4. Power transfer function of the dc blocking stripline capacitor.

In Fig. 4, an obvious decrease in transferred power is observed at frequencies $\mathrm{n} \times 109 \mathrm{GHz}$, where $\mathrm{n}$ is an integer, corresponding to 'geometrical' standing waves of length $n \lambda / 2$. These frequencies correspond to the regions designated by arrows in Figs. 2 and 3, and are in agreement with the LTSEM measurements [4].

The LTSEM results also showed standing waves at most frequencies, but they were more pronounced for example in the range around $170 \mathrm{GHz}$, where no coherent emission was detected (see Fig. 2), although in part of this range some incoherent power was observed (see Fig. 3). The standing wave patterns result from an impedance mismatch between the blocking capacitor $\left(\mathrm{Z}_{\text {out }} \approx 1.0 \Omega\right)$ and the stripline connecting to the detector junction $(Z \approx 5.7 \Omega)$ resulting in $50 \pm 5 \%$ reflection $[3,4]$. In the frequency range around $170 \mathrm{GHz}$, the standing waves are thought to extend from the stripline capacitor into the array. A standing wave partially in the array might change the relative phases of consecutive rows of junctions giving rise to incoherent emission. Also near this frequency range, the array voltage switched between two or more different voltage states and was sometimes accompanied by hysteresis. The effects of such a standing wave on the array dynamics are very complex and require further study.

\section{CONCLUSIONS}

These results provide significant evidence that the high coherent power emitted from 2D arrays of Josephson oscillators is not caused by resonances in the blocking capacitor, but rather by an internal resonance in the array unit cells. Reflected power from the coupling circuit impedance mismatch might provide a feedback mechanism that induces phase-locking in the array. However, preliminary results suggest that the geometrical standing waves totally reflect the array emission from the detector junction and at other frequencies, as in the range around $170 \mathrm{GHz}$, standing waves may even destroy coherence in the array. LTSEM is a useful tool to investigate integrated microwave Josephson circuits. For example, standing waves due to the finite length of the stripline capacitor, and due to an impedance mismatch were observed with LTSEM.

\section{ACKNOWLEDGEMENTS}

The authors thank C. A. Hamilton and R.L. Kautz for many refreshing and stimulating discussions, and the use of their computer programs. This research was supported in part by the Office of Naval Research under contract No. N0001492-F-00040. One of the authors (PAAB) acknowledges the support of the University of Twente in The Netherlands.

\section{REFERENCES}

[1] S. P. Benz and C. J. Burroughs, "Coherent emission from two-dimensional Josephson junction arrays," Appl. Phys. Lett., vol. 58, pp. 2162-2164, May 1991.

[2] S. P. Benz and C. J. Burroughs, "Two-dimensional arrays of Josephson junctions as voltage-tunable oscillators," Supercond. Sci. Technol, vol. 4, pp. 561-567, 1991.

[3] A. Barone and G. Paterno, "Physics and applications of the Josephson effect," New York, 1982, chapter 11.

[4] T. Doderer, D. Hoffmann, R. P. Huebener, N. Kirchmann, C. A. Krulle, S. Lachenmann, D. Quenter, J. Schmidt, S. Stehle, J. Niemeyer, R. Pöpel, S. P. Benz and P. A. A. Booi, "SUSAN (SUperconducting Systems ANalysis) by Low Temperature Scanning Electron Microscopy (LTSEM)," paper EOD-2 submitted to this conference (ASC'92).

[5] C. A. Hamilton, "High-speed, low-crosstalk chip holder for Josephson integrated circuits," IEEE Trans. Inst. Meas., vol. IM-31, pp. 129-131, June 1982.

[6] W. H. Chang, "Numerical calculation of the inductances of a multi-superconductor transmission line system," IEEE Trans. Magn., vol. MAG-17, pp. 764-766, January 1981. 